\title{
Sensory perception during movement in man
}

\author{
C.E. Chapman, M.C. Bushnell, D. Miron, G.H. Duncan, and J.P. Lund \\ Centre de Recherche en Sciences Neurologiques, Ecole de Réadaptation and Faculté de Médecine Dentaire, Université de Montréal, \\ PO Box 6128, Station A, Montréal, Québec H3C 3J7, Canada
}

Summary. The ability of subjects to perceive innocuous stimuli in the presence and absence of movement was evaluated using electrical stimulation of the skin. The subjective intensity of suprathreshold stimuli was unchanged during movement. Discrimination of small differences in the intensity of suprathreshold stimuli (difference thresholds) was also not altered by movement while, in the same subjects, detection thresholds were increased during movement of the stimulated arm. These results suggest that the elevation of detection thresholds during movement can be explained by masking. Both active and passive movement of the stimulated limb increased detection thresholds, with active movement having a slightly greater and more consistent effect than passive movement. Thus, both central and peripheral feedback factors appear to play a role in diminishing one's ability to detect weak stimuli during movement. Attention was also shown to influence performance of the detection task.

Key words: Cutaneous - Somatosensory discrimination - Active movement - Passive movement - Man

\section{Introduction}

Animal and human experiments show that there is a reduction in the transmission of sensory information to the thalamus and somatosensory cortex associated with movement. The amplitude of evoked potentials in the lemniscal system decreases prior to and during voluntary limb movements in cats (Ghez and Lenzi 1971; Ghez and Pisa 1972; Coulter 1974), monkeys (Dyhre-Poulson 1978; Chapman et al. 1984) and man

Offprint requests to: C.E. Chapman, Centre de Recherche en Sciences Neurologiques, Université de Montréal, PO Box 6128, Station A, Montréal, Québec, H3C 3J7, Canada
(Giblin 1964; Coquery and Vitton 1972; Lee and White 1974; Hazemann et al. 1975; Papakostopoulos et al. 1975; Rushton et al. 1981; Starr and Cohen 1985). In keeping with these findings, psychophysical experiments have shown that the threshold for detecting cutaneous stimuli rises when the stimulated area is actively moved (Coquery et al. 1971; Garland and Angel 1974; Dyhre-Poulson 1978; Angel and Malenka 1982) and this change can precede the onset of movement (Coquery 1978). These observations lead to the assumption that sensory perception becomes less acute during movement. But it is not known how, or even whether, the perception of suprathreshold stimuli is modified during movement.

This study examined the ability of subjects to perceive stimuli during arm movement. The experiments, were designed to differentiate between two hypotheses, shown schematically in Fig. 1, which could explain the effects of movement on sensory perception. With the parallel curve hypothesis (Fig. 1A), we postulate that the entire stimulus-response function may be shifted horizontally to the right during movement. In this case, the elevated detection threshold would be associated with a uniform decrease in the subjective intensity of a stimulus during movement. However, since the slope of the stimulus-response function is unchanged, the perceived difference between stimuli would be preserved and thus the difference thresholds would be unchanged during movement. Our second hypothesis is that only the lower part of the curve is displaced (Fig. 1B). In both the somatosensory and the auditory systems, the detection threshold is increased in the presence of a second "masking" stimulus (see, for eg., Craig 1972, 1978; Moody et al. 1976). While the subjective intensity of auditory stimuli limited to the lower end of the intensity range is diminished during the masking procedure, the two stimulus-response curves join at a certain suprathreshold intensity so 

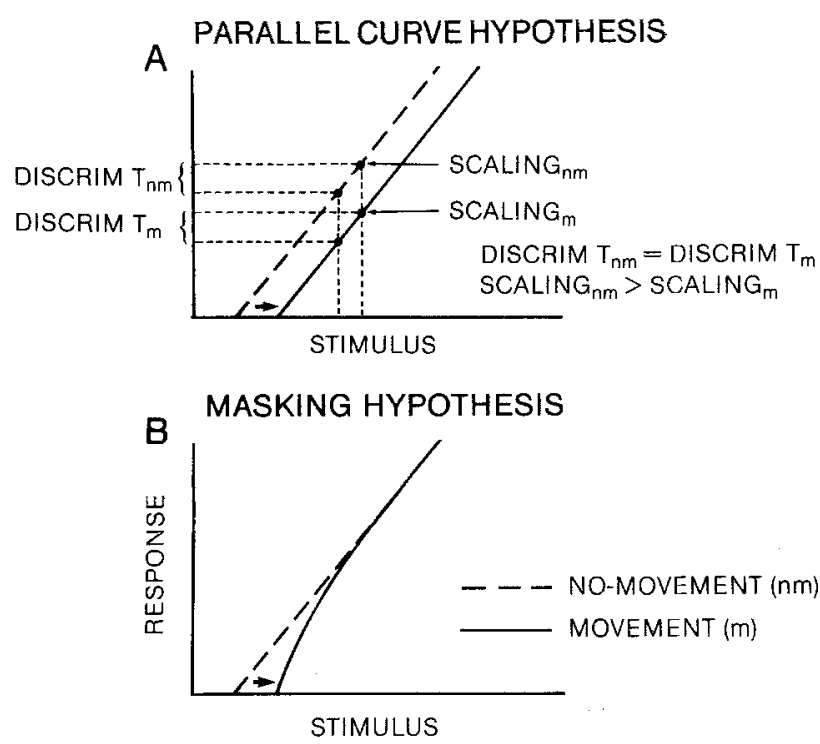

Fig. 1A, B. Hypothetical effects of movement on the perception of innocuous cutaneous stimuli, given that the detection threshold is increased during movement. For both $\mathbf{A}$ and $\mathbf{B}$, the broken line represents the stimulus-response function at rest; the solid line represents the function during movement. A Parallel curve hypothesis. The entire stimulus-response function may be shifted horizontally to the right during movement. B Masking hypothesis. The origin of the stimulus-response curve is shifted to the right during movement but the curves rejoin at a suprathreshold level. At intermediate intensities of stimulation, the slope of the stimulus-response curve is increased

that, above that level, subjective intensity is not altered in the presence of the masking stimulus (Pohlman and Kranz 1924; Fowler 1937). With respect to the present study, if the signals associated with movement - efference copy and/or peripheral reafference associated with the movement - act as masking stimuli, and if the testing occurs in the range over which the two curves are identical, then one might expect that the elevated detection threshold would be associated with no change in either subjective intensity or in the difference threshold. Testing below the level at which the two curves join would be expected to diminish the subjective intensity and increase the difference threshold (increase in slope, see Fig. 1B.)

In order to differentiate between our two hypotheses, we evaluated subjects' ability to perform three different perceptual tasks in the presence and absence of movement. First, their ability to distinguish between suprathreshold stimuli was evaluated (difference threshold). Second, the perceived intensity of stimuli was examined in a scaling task (magnitude estimation). Finally, the ability of those same subjects to detect threshold stimuli during movement and at rest was measured. The results show that neither the ability to discriminate between supra- threshold stimuli, nor the ability to estimate the subjective magnitude of suprathreshold stimuli, are altered during movement. It is suggested that the diminished ability to detect threshold stimuli can be explained by a masking effect. A preliminary report of some of these data has been presented elsewhere (Lund et al. 1986).

\section{Methods}

Subjects were trained to perform three tasks - a detection task, a discrimination task and a scaling task - under various experimental conditions. All tasks used a method of constant stimuli (Woodworth and Schlosberg 1954) in which 9 different intensities of electrical stimulation (single, constant current, square wave pulses of $2 \mathrm{~ms}$ duration) were presented in a quasi-random fashion via surface electrodes ( $7 \mathrm{~mm}$ diameter, cathode proximal) applied to the mid-ventral aspect of the right forearm. The electrodes were not placed in close proximity to any major cutaneous nerve trunk.

\section{Detection task}

The absolute threshold ( $50 \%$ detection level) was estimated, with the subject at rest, at the beginning of the session (range 0.23 to $1.11 \mathrm{~mA}$ ). Then, nine intensities of current, distributed linearly around this value $(10-50 \mu \mathrm{A}$ steps in different subjects, $25 \mu \mathrm{A}$ for most subjects; 4 above, 4 below and 1 equal), were presented to the subject at unpredictable intervals, in a random sequence during movement and at rest (see Experimental design, below). The subject was asked to report the occurrence of each perceived stimulus.

\section{Discrimination task}

Pairs of stimuli, consisting of the standard stimulus followed by the comparison stimulus (delay about $1 \mathrm{~s}$ between the standard and the comparison), were delivered in a quasi-random fashion to the subject at times not predictable by the subject. A clearly detectable but innocuous stimulus served as the standard stimulus (range 0.75 to $4.1 \mathrm{~mA}$ in different subjects) in this task. The subjects were asked to report if the comparison stimulus was the same or different from the preceding standard pulse. The discrimination threshold was estimated at the beginning of the session $(50 \%$ called different) with the subject at rest (range 0.3 to $1.1 \mathrm{~mA}$ in 7 subjects and 1.1 to $2.1 \mathrm{~mA}$ in 1 subject) and nine intensities distributed in equal steps about this value were chosen as the comparison stimuli (0.075 to $0.6 \mathrm{~mA}$ steps in different subjects; 4 above, 4 below and 1 equal). The comparison stimulus of the lowest intensity was the same as the standard, to control for possible false alarms.

\section{Scaling task}

For this task, the lowest detectable and the highest, non-painful currents were estimated at the beginning of the session, with the subject at rest. Nine currents distributed linearly (equal steps varying from 0.15 to $1.1 \mathrm{~mA}$ ) about a value lying midway between the two extremes, and covering $75 \%$ of this range, were chosen (range 0.8 to $9.4 \mathrm{~mA}$ ). All stimuli were clearly detectable but innocuous. Care was taken to ensure that the range of values tested encompassed the values used for determination of the 
DETECTION TASK
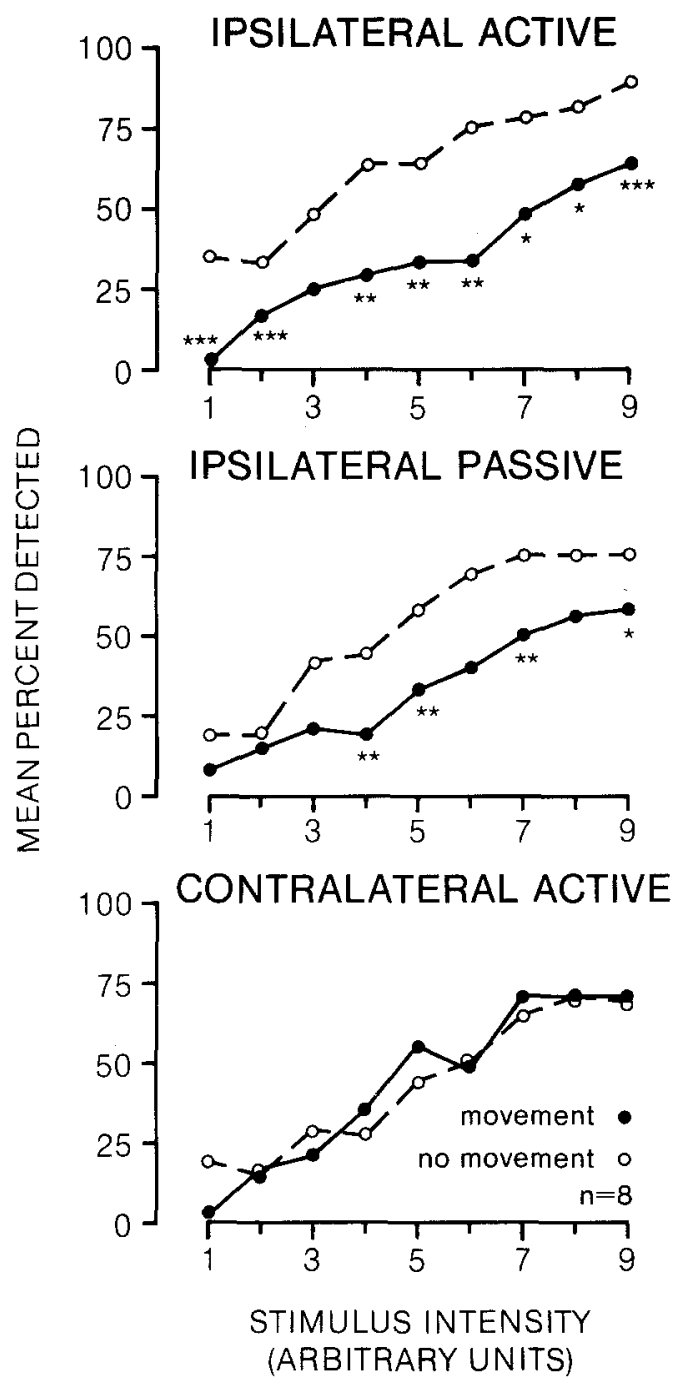

Fig. 2. Detection task. Percent of trials in which subjects detected the stimulus, with (O) and without $(O)$ movment, in three conditions: active and passive movement of the ipsilateral, stimulated arm and active movement of the contralateral, nonstimulated arm. The data from 8 subjects have been pooled. Each movement condition was tested in a separate session. Each point is an average of the total number of stimuli detected at a particular intensity, ranging from the lowest ( 1 arbitrary unit) to the highest ( 9 arbitrary units) intensity used. For each of the three movement conditions, the performance of the subjects at the 9 individual intensities of stimulation was compared during the movement and no-movement conditions (Wilcoxon matched-pairs, signed-ranks test $-{ }^{* * *} P<0.005$; $^{* *} P<0.025$; ${ }^{*} P<0.05$ )

difference threshold. Prior to the start of the scaling task (subject at rest), 3 stimuli were presented to the subject - one low intensity, one high intensity and finally the mid-range value. The subject was asked to assign any number which seemed appropriate to the last stimulus (the mid-range value). The subjects were then instructed to indicate the intensity of the subsequent stimuli, given at unpredictable intervals, by assigning numbers to them, proportional to their subjective impression.

\section{Movement conditions}

The detection, discrimination and scaling tasks were each performed at rest and during movement. In all, 3 different movement conditions were investigated: active and passive movement of the stimulated arm and active movement of the contralateral, nonstimulated arm. The subject was seated with the arms resting on two independent manipulanda. For the arm which was not stimulated, the forearm was supported at the wrist and the elbow. For the stimulated arm, the proximal point of support was shifted because pilot experiments showed that when the elbow rested on the rotating platform, there was considerable movement of the neighbouring skin. In order to minimize movement of the electrodes, the proximal support was therefore given by a sling under the upper arm. Elbow position was monitored with potentiometers and displayed to the subject on an oscilloscope. The subjects were trained to perform a repetitive flexion and extension of the elbow, ipsi- or contralateral to the stimulating electrodes, moving between 2 target lines on the oscilloscope. The amplitude of the movement was approximately 60 degrees, at a pace $(0.5 \mathrm{~Hz})$ indicated by a series of clicks. The subjects were instructed to make smooth, sinusoidal movements between the two target lines so that the arm was in almost continuous motion. Smooth, passive, sinusoidal movements between the same target lines were applied manually by an experimenter, using an extension attached to the back of the manipulandum. The frequency of movement was signalled in exactly the same manner as for the active trials so that, as far as possible, there were no systematic differences between active and passive movement. Stimuli were given without regard to the phase of movement.

\section{Experimental design}

A total of five female and six male volunteers participated in this study. Two series of experiments were performed. In the first series, eight subjects participated in three experimental sessions, one session for each movement condition (ipsilateral active, ipsilateral passive and contralateral active). All three tasks (detection, discrimination and scaling) were performed in each of these sessions. The order of testing was counterbalanced within and between subjects. For the detection and discrimination tasks, there were six alternating blocks of 9 movement and 9 nomovement trials. For the scaling task, the protocol was slightly modified so that each movement trial was followed by a nomovement trial. In this manner we hoped to reduce the possibility that the strategy used by the subject to estimate the intensity of the stimuli changed between the two conditions.

In the second series of experiments, the effects of the three movement conditions on the performance of the detection task were evaluated within the same session to permit a direct comparison of results obtained under identical recording conditions. Six subjects participated in one experimental session. The same protocol of six alternating blocks of nine movement and nine no-movement trials for each movement condition was employed. The order of testing was counterbalanced between subjects.

\section{Results}

Figure 2 and Table 1 show the results obtained from eight subjects in the detection task (first series). The average stimulus-response curves during movement and in the absence of movement are shown for each of the three conditions. Inspection of the graphs 
Table 1. Summary of the data $(n=8$ subjects)

\begin{tabular}{|c|c|c|c|c|c|c|c|c|c|}
\hline & \multicolumn{3}{|c|}{ Detection task } & \multicolumn{3}{|c|}{ Discrimination task } & \multicolumn{3}{|c|}{ Scaling task } \\
\hline & $\begin{array}{l}\text { Ipsi } \\
\text { active }\end{array}$ & $\begin{array}{l}\text { Ipsi } \\
\text { passive }\end{array}$ & $\begin{array}{l}\text { Contra } \\
\text { active }\end{array}$ & $\begin{array}{l}\text { Ipsi } \\
\text { active }\end{array}$ & $\begin{array}{l}\text { Ipsi } \\
\text { passive }\end{array}$ & $\begin{array}{l}\text { Contra } \\
\text { active }\end{array}$ & $\begin{array}{l}\text { Ipsi } \\
\text { active }\end{array}$ & $\begin{array}{l}\text { Ipsi } \\
\text { passive }\end{array}$ & $\begin{array}{l}\text { Contra } \\
\text { active }\end{array}$ \\
\hline No-movement & 34.5 & 32.5 & 21.0 & 22.5 & 23.0 & 22.5 & 545 & 490 & 510 \\
\hline Movement & $19.5^{* *}$ & $24.0^{*}$ & 23.5 & 23.0 & 23.0 & 22.0 & 513 & 499 & 496 \\
\hline
\end{tabular}

Detection task. Median number of stimuli detected (54 stimuli delivered during, respectively, the movement and no-movement conditions). Discrimination task. Median number of stimuli called different (54 pairs of stimuli delivered during, respectively, the movement and nomovement conditions: 48 of the pairs were different). Scaling task. Median summed score (normalized) is reported (maximum 900 for each of the movement and no-movement conditions). "Movement" and "No-movement" performance is compared for each condition (Wilcoxon matched-pairs, signed-ranks test) and the significance of any difference is shown. ${ }^{*} P<0.025 ;{ }^{* *} P<0.005$

indicates that movement of the ipsilateral arm, active and passive, elevated the detection threshold $(50 \%$ detection level). The group thresholds, measured from the curves shown in Fig. 2, were 2.3 and 1.6 times greater during, respectively, active and passive ipsilateral movement. Neither during the movement nor the no-movement conditions did the subjects report stimuli when they were not given (false alarms). The results of separate, paired comparisons of the number of stimuli detected at the individual stimulus intensities during the movement and nomovement conditions (Wilcoxon matched-pairs, signed-ranks test) are also shown in Fig. 2. Significantly fewer stimuli were detected at 8 of 9 intensities tested during active movement as compared to only 4 of 9 intensities during passive movement. Thus, active movement appeared to have a slightly greater effect than did passive movement of the stimulated arm.

In contrast to movement of the ipsilateral arm, there was no obvious difference between the subjects' performance during contralateral arm movement and during the corresponding no-movement trials. Inspection of Fig. 2 (bottom) shows that the two curves are virtually identical. Statistical comparisons (as above) of the subjects' performance during contralateral movement and during the corresponding no-movement trials failed to show any significant changes. However, when the number of stimuli detected was summed across all intensities of stimulation (median values given in Table 1), it was noted that the number of stimuli detected during the no-movement trials was significantly lower than for either of the two other conditions (Wilcoxon, $P<$ 0.025). The possible importance of this observation could not be assessed as the movement conditions were tested in separate sessions (see below) and so the range of intensities tested was not necessarily comparable.

In order to compare results obtained on different days, it was important to demonstrate that the stimulating conditions were similar. We therefore compared the absolute threshold values $(50 \%$ detection level) for each subject at rest in the three sessions (Friedman two-way analysis of variance by ranks). This analysis indicated that no significant changes occurred.

Figure 3 and Table 1 show the results of the same 8 subjects in the discrimination task. Although the intensitiy of the standard varied slightly from session to session in individual subjects, there was no significant change in the intensity of the standard stimulus employed in the three sessions (Friedman two-way analysis of variance). Furthermore, for all subjects the tendency to report a difference when there was none was low and was the same in all conditions $(0$ difference in Fig. 3), implying that the subjects were performing the task as instructed. Inspection of these graphs shows that performance of the discrimination task was virtually uninfluenced by any of the three movement conditions. The group thresholds $(50 \%$ called different) during movement, measured from the curves in Fig. 3, did not vary by more than $6 \%$ from the values during the no-movement trials and none of the indiviual, paired comparisons showed a significant change during any of the three movement conditions. Thus the results suggest that the ability to discriminate between suprathreshold innocuous stimuli is not altered by movement. The data were also examined to see if there might have been some systematic differences in the results when the intensity of the standard was closer to the absolute threshold. Some slight indication of an increase in the difference threshold was seen during ipsilateral active movement in those subjects with the lowest standard stimulus $(\mathrm{n}=4)$ but the difference was significant in only one subject (Wilcoxon, $P<0.05$ ). No consistent changes were seen in the other two movement conditions.

The results of the scaling task $(n=8)$ are shown in Fig. 4 and Table 1. The individual subjective scales were normalized in order to pool the data. In this 
DISCRIMINATION TASK

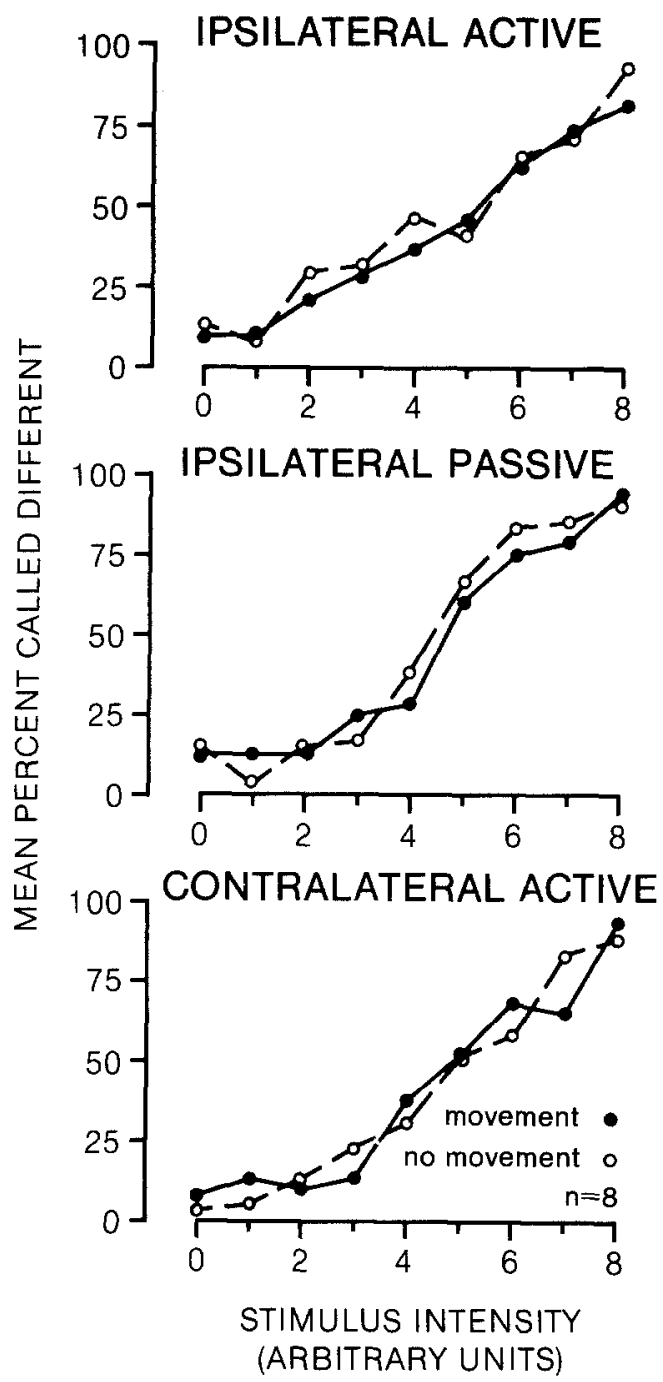

Fig. 3. Discrimination task. Percent of trials in which subjects reported that the test stimulus was different from the preceding standard stimulus $(n=8)$ as a function of the intensity of the test stimulus (test $=$ standard at intensity 0 ). Plotted as in Fig. 2 for the three conditions (with and without movement)

task, the intensities tested always included those used in the discrimination task in the same session. Although the tested range varied somewhat from session to session, for the individual subjects there was no significant change in the intensities tested in the three sessions (Friedman two-way analysis of variance). Examination of Fig. 4 shows that none of the three movement conditions had an effect on the ability of subjects to estimate the intensity of the stimulation. The two paired curves, movement and no-movement, are virtually identical for each of the conditions tested. The paired comparison of the subject's estimates at the 9 levels of stimulation

\section{SCALING TASK}

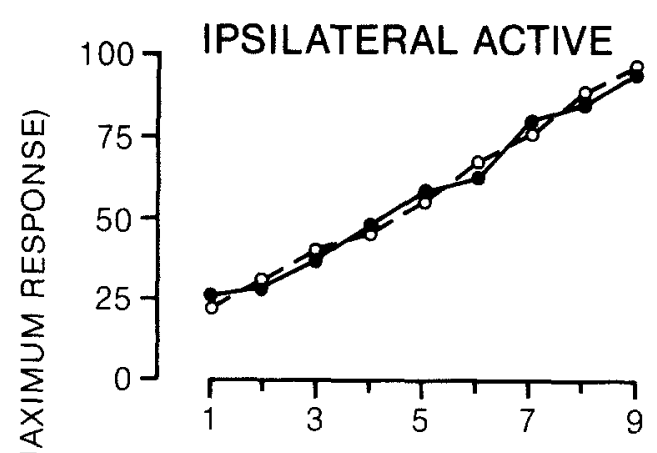

$\sum 1007$ IPSILATERAL PASSIVE
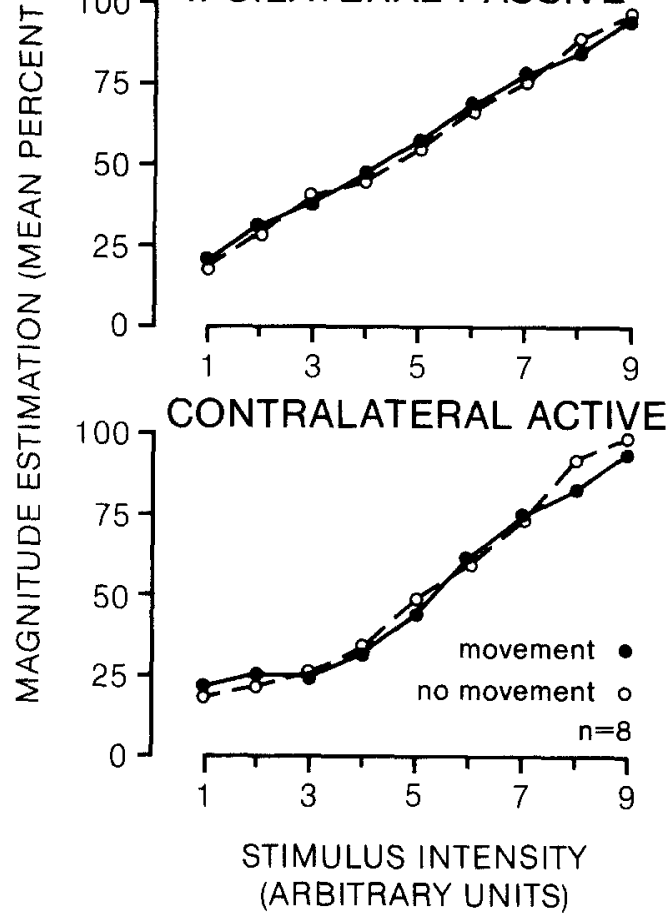

Fig. 4. Scaling task. The effects of the three movement conditions on the apparent intensity of suprathreshold stimuli were evaluated. The data from 8 subjects are pooled. Plotted as in Fig. 2

tested showed that there were no significant changes. Likewise, the total scores (median values reported in Table 1) showed no significant change. Finally the data were examined to see if there were some systematic differences between subjects related to the intensities of stimulation tested (close to or well above the absolute threshold). No trends were observed in any of the three movement conditions. Thus movement appears to have no effect on the ability of subjects to estimate the subjective intensity of an innocuous electrical shock, over the ranges tested in this study.

In a second series of experiments, the effects of the three movement conditions on the performance of the detection task were evaluated in the same 


\section{DETECTION TASK}
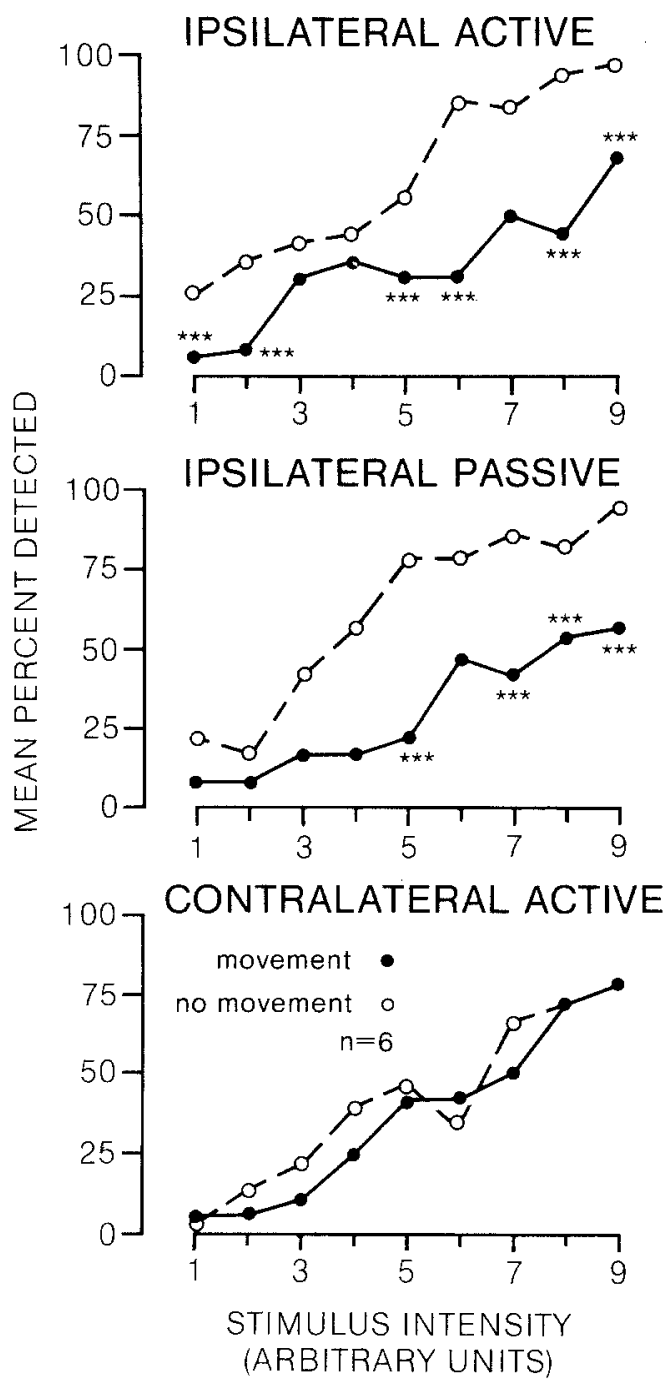

Fig. 5. Detection task. In a single session (6 subjects), the effects of the three movement conditions on performance of the detection task were evaluated. Plotted as in Fig. 2. Significant changes in performance at some of the 9 individual intensities of stimulation were observed $\left({ }^{* * *} P<0.005\right)$

session in order to permit a more direct comparison of the results. Two issues were addressed in this series of experiments. First, the results from the initial series of experiments, which were obtained in three separate sessions under similar but not identical recording conditions, suggested that active movement of the stimulated arm produced a more consistent elevation of the absolute threshold than did passive movement. Second, the median number of stimuli detected at rest for the contralateral movement condition was significantly lower than for either of the other two movement conditions (Table 1 and
Table 2. Median number of stimuli detected during the three movement conditions ( $\mathrm{n}=6$ subjects) tested in a single session

\begin{tabular}{|c|c|c|c|}
\hline & $\begin{array}{l}\text { Ipsi } \\
\text { active }\end{array}$ & $\begin{array}{l}\text { Detection task } \\
\text { Ipsi } \\
\text { passive }\end{array}$ & $\begin{array}{l}\text { Contra } \\
\text { active }\end{array}$ \\
\hline No-movement & 36.0 & 33.5 & 20.5 \\
\hline Movement & $18.0^{* *}$ & $16.0^{*}$ & 20.5 \\
\hline
\end{tabular}

"Movement" and "No-movement" are compared for each condition (Wilcoxon matched-pairs, signed-ranks test) and the significance of any difference is shown. ${ }^{*} P<0.025$; ${ }^{* *} P<0.005$. A total of 54 stimuli were delivered, respectively, during the movement and the no-movement trials

above) but this effect was specific to the detection task. There was a significant change in performance in the no-movement conditions for the detection task (Friedman two-way analysis of variance, $P<0.03$ ) but not for either the discrimination or the scaling tasks.

The results of this second series of experiments, shown in Fig. 5 and Table 2, were almost identical to those obtained in the first series. Both active and passive ipsilateral movement elevated the detection threshold, with active movement again elevating the detection threshold more than passive movement. For example, the results of the paired comparison (Fig. 5), now using exactly the same intensity of stimulation for all conditions, showed that significantly fewer stimuli were detected at more of the intensities of stimulation tested during active movement, 6 , than during passive movement, 4 . Furthermore, while there was a significant decrease in the total number of stimuli detected with both active and passive movement of the stimulated arm, the level of significance was greater for active $(P<0.005)$ than for passive movement $(P<0.025)$. Similar results were observed in the first series of experiments (Table 1).

As regards the contralateral movement condition, there was once again no effect when comparing the performance during movement with that at rest (Fig. 5, bottom and Table 2). But, as in the original task, the number of stimuli detected by the subjects at rest was again significantly lower than for either of the two other movement conditions (Wilcoxon, $P<$ 0.025 ) using exactly the same intensity of stimulation. In contrast, during movement in this and in the first series of experiments, there was no difference in the number of stimuli detected in the three conditions (Friedman two-way analysis of variance by ranks), i.e. the detection threshold was elevated as much during contralateral testing, both at rest and during movement, as during ipsilateral movement. 


\section{Discussion}

The results of these experiments show that the perception of suprathreshold stimuli is not altered during movement and thus support the second of our two hypotheses, the masking hypothesis (Fig. 1B). The validity of our method of testing (a method of constant stimuli, Woodworth and Schlosberg 1954) was ensured by showing that, using the same method, the detection of weak electrical shocks was diminished during movement of the stimulated arm, confirming the results of previous investigators (Coquery et al. 1971; Garland and Angel 1974; Dyhre-Poulson 1978; Angel and Malenka 1982). One could argue that the failure to observe a change in the discrimination task was a function of the method of testing: only in this task was the test stimulus preceded by a comparison stimulus which may have served as a warning cue. The fallacy in this argument is, however, that the subject had to make a comparison between the stimuli, i.e. both stimuli had to be attended to and perceived. While it is difficult to completely exclude the possibility that the increase in the detection threshold is attributable to a shift in the subjects' response criteria during movement, the failure to observe the same effect during movement of the contralateral, nonstimulated arm would argue against such an interpretation of the results. Two observations indicate that the elevation of detection thresholds during ipsilateral movement is a specific effect rather than a nonspecific decrease of sensory perception during movement. First, contralateral movement had no additional effect upon the detection threshold and second, ipsilateral active movement had slightly greater effects than ipsilateral passive movement.

The observation that the subjective intensity of the stimulus was also unchanged was taken to suggest that the stimulus-response function was the same during both the movement and the no-movement trials. Although there was considerable variation in the values tested for the scaling task in different subjects, the range of intensities used always encompassed those used in the discrimination task and these were largely restricted to stimulus intensities of 2 through 5 arbitrary units (Fig. 4). Thus over this part of the stimulus-response function there was (i) no change in slope and (ii) no change in subjective intensity, i.e. the two curves, movement and nomovement, were identical. We did not determine the extent of the masking effect but it was evidently restricted to the very lowest intensities of stimulation, lower than those used for the scaling task.

With respect to the masking hypothesis, the absence of any effect in the discrimination task is taken as evidence that the slope of the stimulusresponse function is unchanged by movement, at least over the range of values tested. According to this hypothesis, however, one would have predicted that the discrimination threshold would be increased at the low end of the scale. There was some indication of this during ipsilateral active movement but the difference was significant in only one subject. Indeed, the importance of the latter observation is questionable as there was no such change associated with passive movement, as would have been expected from the results of the detection task. Clearly further experiments are required to determine the range over which the stimulus-response curve is nonlinear.

It has been reported that cortical somatosensory evoked potentials are decreased in amplitude during movement (Giblin 1964; Coquery et al. 1972; Coquery and Vitton 1972; Hazemann et al. 1975; Papakostopoulos et al. 1975; Rushton et al. 1981; Chapman et al. 1984; Starr and Cohen 1985). One might speculate that if subjective intensity is determined on the basis of the central neural code then, when neural discharge is diminished, there would be a parallel decrease in the subjective intensity of the stimulus. How can we reconcile these observations with our data which show that subjective intensity is not reduced? Two comments can be made. First, in the majority of the evoked potentials studies, the test stimulus had no significance for the subject, i.e. the subject was not actively discriminating the stimulus. In the one study in which the effects of movement on cortical somatosensory evoked potentials were investigated during the performance of a detection task, Coquery et al. (1972) only showed that a late component of the cortical evoked potential (their wave 3) was diminished in amplitude during movement. The significance of this observation is unclear because the source of these late waves is unknown (Rushton et al. 1981). One can speculate that in a situation in which the stimulus is actively discriminated there might be some recalibration of the perceptual processes to take into account the state of the animal. Evidence that such mechanisms exist is obtained from studies of the effects of attention on perception and on neuronal responsiveness to peripheral stimuli. Thus, it is known that attention to a somatosensory stimulus improves its detectability (Bushnell et al. 1985) and that attention can modify the responses of parietal cortical neurones to somatic stimuli, even within the primary somatosensory cortex (Hyvärinen et al. 1980; Nelson 1984). Secondly, it is not clear if any relationship between the amplitude of cortical evoked potentials and the perceived magnitude of a stimulus exists. Drowsiness is accom- 
panied by a large increase in the amplitude of the somatosensory cortical evoked potentials but, paradoxically, a reduced response of parietal association neurones to somatic stimuli (Mountcastle et al. 1975), as well as reduced attentiveness to somatic stimuli. In order to resolve this problem, further experiments should test the effects of movement upon central responses to somatic stimuli while the subject is actively discriminating the stimulus.

The origin of the masking effect remains obscure. The present results do, however, support the proposal that both central and peripheral factors contribute to the elevation of detection thresholds during movement. Peripheral factors were certainly important since passive movements were effective in raising the detection threshold in this study. We cannot entirely exclude the possibility that minimal dislocations of the surface electrodes may have contributed to the observed effect, but every effort was made to diminish any shearing forces about the elbow by modifying the point of support of the arm on the stimulated side (Methods). We therefore conclude that peripheral feedback from the moving limb may have acted to diminish transmission from the periphery to higher centres. These results are difficult to reconcile with those of Ghez and Pisa (1972) and Coulter (1974), who found that lemniscal potentials elicited by stimulation of a cutaneous nerve in the cat were unchanged by vigorous passive movements. They noted, however, that tactile stimulation did reduce lemniscal evoked potentials. The differences between the present results and the animal studies might, therefore, be reconciled if our passive movements elicited considerably discharge in cutaneous afferents. Indeed, it has been shown that during movement of the digits in man, there is considerable movementrelated discharge in cutaneous mechanoreceptor afferents (Hulliger et al. 1979). Elbow movements stretch the skin overlying the moving joint and probably excite cutaneous afferents.

Central factors probably contribute to the masking effect because active movement more consistently increased detection thresholds than did passive movement. This interpretation is supported by observations that evoked responses in the lemniscal system are reduced prior to the onset of movement, i.e. at a time before any afferent feedback is generated (Ghez and Lenzi 1971; Ghez and Pisa 1972; Coquery and Vitton 1972; Coulter 1974; Dyhre-Poulson 1978; Chapman et al. 1984; Starr and Cohen 1985). Indeed, Coquery (1978) has shown that detection of electrical stimuli applied to the fingertip may be diminished prior to the onset of voluntary flexion of the fingers.

The effects of the contralateral movement condition on detection thresholds were more puzzling.
Previous studies have reported contradictory results. Dyhre-Poulson (1978) found that contralateral movement diminished the ability to detect weak stimuli while Coquery et al. (1972) and Garland and Angel (1974) found that there was no effect. In the present study, we observed that the detection threshold rose when the subject knew that movements of the contralateral arm were required in the next block of trials. However, there was no further change during contralateral movement (Tables 1 and 2). This modulation was only observed for the detection task. Since the control trials for the three movement conditions were performed under identical conditions, the increase in the absolute threshold during the nomovement trials for the contralateral movement condition can probably be attributed to an attentional effect. The subjects' attention was almost certainly diverted to the contralateral arm during the associated movement trials. This suggests that attention may be a more critical factor for absolute threshold determination than for either difference thresholds or magnitude estimation.

Since some of the modulation is likely to arise from central sources, a good candidate would be the sensorimotor cortex. This structure exerts control over sensory transmission at different levels of the lemniscal system and, moreover, the controls may vary with the modality of the input (Tsumoto et al. 1975). In the present study, the electrical stimulation undoubtedly activated, nonspecifically, afferents from a number of different cutaneous receptor types (presumably only A beta afferents as the sensation was never painful). It would be interesting to determine the effects of movement on the perception of different somatosensory submodalities in future experiments.

Acknowledgements. We thank R. Bouchoux, M. Boyer and D. Veilleux for technical assistance. Drs. T. Drew, J.F. Kalaska and $\mathrm{Y}$. Lamarre are thanked for their comments on early versions of this manuscript. Supported by the Medical Research Council of Canada and the Fonds de la Recherche en Santé du Québec (FRSQ). C.E. Chapman is a Chercheur-Boursier of the FRSQ.

\section{References}

Angel RW, Malenka RG (1982) Velocity-dependent suppression of cutaneous sensitivity during movement. Exp Neurol 77: 266-274

Bushnell MC, Duncan GH, Dubner R, Jones RL, Maixner W (1985) Attentional influences on noxious and innocuous cutaneous heat detection in humans and monkeys. $J$ Neurosci 5: $1103-1110$

Chapman CE, Rispal-Padel L, Parent MT, Lamarre Y (1984) Gating of lemniscal input at thalamic and cortical levels during conditioned arm movements in the monkey. Abstr Soc Neurosci 10: 735 
Coquery JM (1978) Role of active movement in control of afferent input from skin in cat and man. In: Gordon G (ed) Active touch. Pergamon Press, Oxford, pp 161-169

Coquery JM, Coulmance M, Leron MC (1972) Modifications des potentiels évoqués corticaux somesthésiques durant des mouvements actifs et passifs chez l'homme. Electroenceph Clin Neurophysiol 33: 269-276

Coquery JM, Malcuit G, Coulmance M (1971) Altérations de la perception d'un stimulus somesthésique durant un mouvement volontaire. C R Soc Biol (Paris) 165: 1946-1951

Coquery JM, Vitton N (1972) Altérations des potentiels évoqués sur le cortex somesthétique du chat durant un movement conditionné. Physiol Behav 8: 963-967

Coulter JD (1974) Sensory transmission through lemniscal pathway during voluntary movement in the cat. J Neurophysiol 37: $831-845$

Craig JC (1972) Difference threshold for intensity of tactite stimuli. Percept Psychophys 11: 150-152

Craig JC (1978) Vibrotactile pattern recognition and masking. In: Gordon G (ed) Active touch. Pergamon Press, Oxford, pp 229-242

Dyhre-Poulson P (1978) Perception of tactile stimuli before ballistic and during tracking movements. In: Gordon G (ed) Active touch. Pergamon Press, Oxford, pp 171-176

Fowler EP (1937) The diagnosis of diseases of the neural mechanism of hearing by the aid of sound well above threshold. Laryngoscope (St Louis) 47: 289-300

Garland HT, Angel RW (1974) Modulation of tactile sensitivity during movement. Neurology (NY) 24: 361

Ghez C, Lenzi GL (1971) Modulation of sensory transmission in cat lemniscal system during voluntary movement. Pflügers Arch 323: 273-278

Ghez C, Pisa M (1972) Inhibition of afferent transmission in cuneate nucleus during voluntary movement in the cat. Brain Res 40: 145-151

Giblin DR (1964) Somatosensory evoked potentials in healthy subjects and in patients with lesions of the nervous system. Ann NY Acad Sci 112: 93-142

Hazemann P, Audin G, Lille F (1975) Effect of voluntary selfpaced movements upon auditory and somatosensory evoked potentials in man. Electroenceph Clin Neurophysiol 39: $247-254$

Hulliger M, Nordh E, Thelin A-E, Vallbo AB (1979) The responses of afferent fibres from the glabrous skin of the hand during voluntary finger movements in man. J Physiol (Lond) 291: $233-249$

Hyvärinen J, Poranen A, Jokinen Y (1980) Influence of attentive behavior on neuronal responses to vibration in primary somatosensory cortex in the monkey. J Neurophysiol 43 : $870-882$

Lee RG, White DG (1974) Modification of the human somatosensory evoked response during voluntary movement. Electroenceph Clin Neurophysiol 36: 53-62

Lund JP, Bushnell MC, Chapman CE, Duncan GH (1986) The detection of differences between suprathreshold stimuli delivered to the skin is not modified by movement. Proc Int Union Physiol Sci 16: 571

Moody DB, Beecher MD, Stebbins WC (1976) Behavioral methods in auditory research. In: Smith CS, Vernon JA (eds) Handbook of auditory and vestibular research methods. Charles C Thomas, Springfield Ill, pp 439-497

Mountcastle VB, Lynch JC, Georgopoulos A, Sakata H, Acuna C (1975) Posterior parietal association cortex of the monkey: command functions for operations within extrapersonal space. J Neurophysiol 38: 871-908

Nelson RJ (1984) Responsiveness of monkey primary somatosensory cortical neurons to peripheral stimulation depends on 'motor-set'. Brain Res 304: 143-148

Papakostopoulos D, Cooper R, Crow HJ (1975) Inhibition of cortical evoked potentials and sensation by self-initiated movement in man. Nature 258: 321-324

Pohlman AG, Kranz FW (1924) Binaural minimum audition in a subject with ranges of deficient acuity. Proc Soc Exp Biol (NY) 21: 335-337

Rushton DN, Rothwell JC, Craggs MD (1981) Gating of somatosensory evoked potentials during different kinds of movement in man. Brain 104: 465-491

Starr A, Cohen LG (1985) 'Gating' of somatosensory evoked potentials begins before the onset of voluntary movement in man. Brain Res 348: 183-186

Tsumoto T, Nakamura S, Iwama K (1975) Pyramidal tract control over cutaneous and kinesthetic sensory transmission in the cat thalamus. Exp Brain Res 22: 281-294

Woodworth RS, Schlosberg H (1954) Experimental psychology. Holt Rinehart and Winston, New York

Received October 14, 1986 / Accepted June 23, 1987 